\title{
Synthetic Bio-Inorganic Materials in Various Artificial Organs
}

\author{
Prof. Virendra Umale \\ Professor, Priyadarshini Indira Gandhi College of Engineering, \\ Nagpur,Maharashtra,India \\ virendraumale@gmail.com
}

Abstract-

Bioinorganic materials play the important role in all living organisms. Mostly these compounds are responsible for the vital actions of an organism such as enzymatic actions, respiration, photosynthesis, metal ion transport etc. In recent time numerous bioinorganic materials have been synthesized partially or completely in the laboratories. These synthetic bioinorganic materials serve as medical boon for mankind as they are the key for development of artificial tissues and organs. In this paper we are discussing about artificial organs and related bioinorganic materials.

Keywords: Artificial organs, bioinorganic materials, polymers etc.

\section{INTRODUCTION}

The organic and inorganic materials of a living organism make an alternate side of material world. Biological materials, for example, polysaccharides, proteins, nucleic acids and lipids have delicate and flexible nature. They show tremendous capacities with high proficiency and particularity. In the combination with synthetic or man-made materials these materials can't be regenerated or recreated easily. The above properties of organic materials assume the prime job in the development of profoundly modern practical frameworks. The most ideal approach to acquire profoundly steady and utilitarian bio materials for their handy applications is to create cross breeds of inorganic and characteristic natural materials. The biologically started materials are efficient and hold numerous important nanostructures.

Consequently the most entrancing methodology in the advancement of an engineered bio polymer is the hybridization of inorganic and biological components in a controlled way. The synthesis of artificial bio materials is an interdisciplinary field which interfaces biotechnology, material science and nanotechnology. Other processes like biominerlization, synthesis and development of bio-inspired materials and biomimetic systems are patrilineal to the synthetic 
artificial bio materials. The recent developments of novel bio nano-composites with multifunctionalities lead us to the next generation in this field.

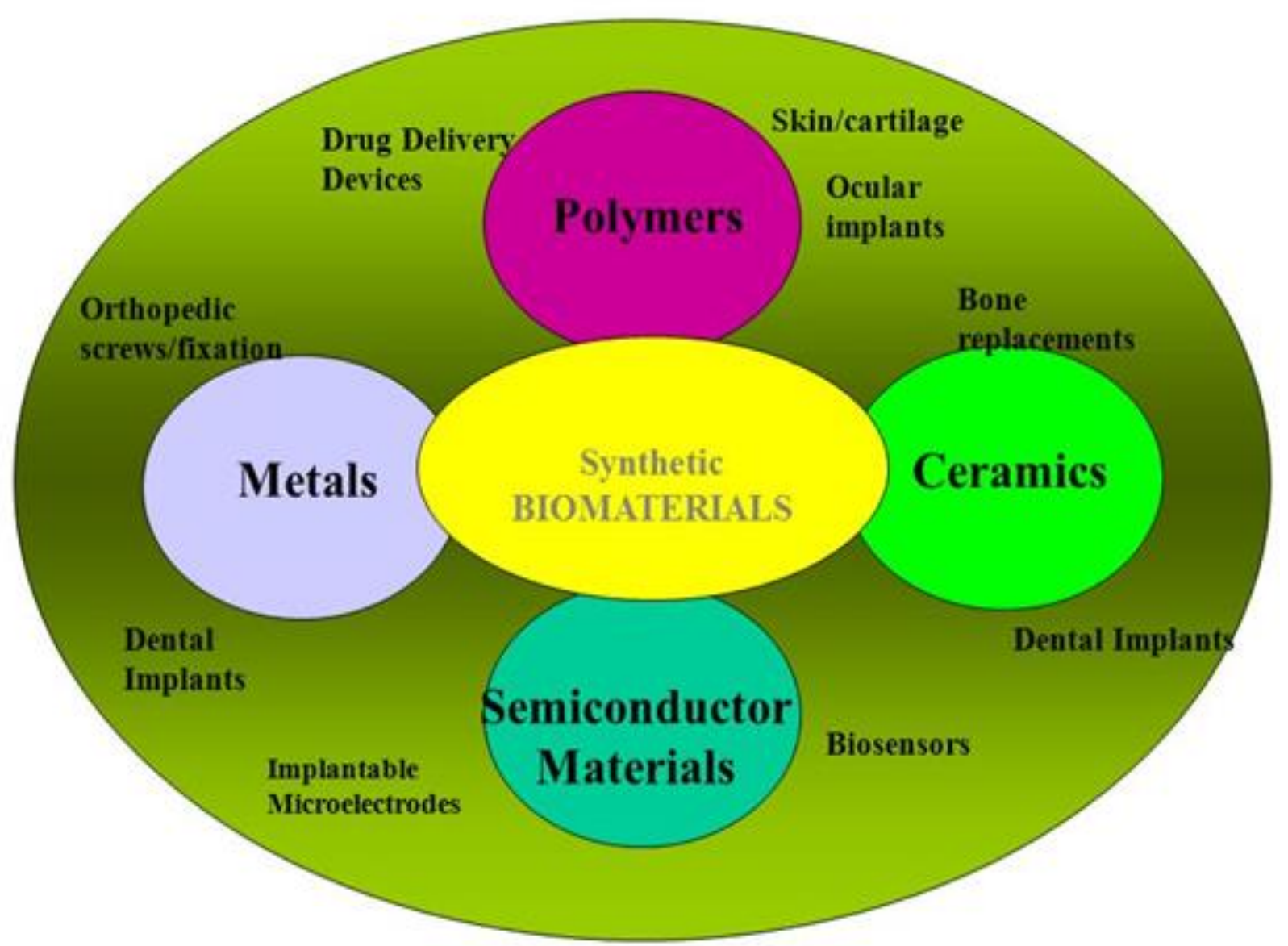

Fig 1: Synthetic Biomaterial

\section{ARTIFICIAL BIO MATERIALS}

Artificial organs are generally defined as any device, machine or complex biological structure which is partially or completely synthetic in nature and that could be implanted or integrated into human body to perform the tasks of a particular biological structure ${ }^{\text {which }}$ has been damaged and should be replaced due to some medical reasons. The scientists are continuously working to procure newer, safer and cheaper pathways to fabricate artificial organs which could curtail the time for organ transplant and transform the surgery. A brief description of sundry artificial organs is as follows:

Artificial Bone:- it is a bone like synthetic material which is used as bone grafts in order to replace the human bones that was lost because of disease or severe fractures. The mutilated bone 
can be replaced by autografts (bone from the other parts of the body), allografts (bone from cadavers), megagrafts (various bioceramics), callografts (hydroxyapatite/tricalcium phosphate and bovine collagen) and metallic alloys.
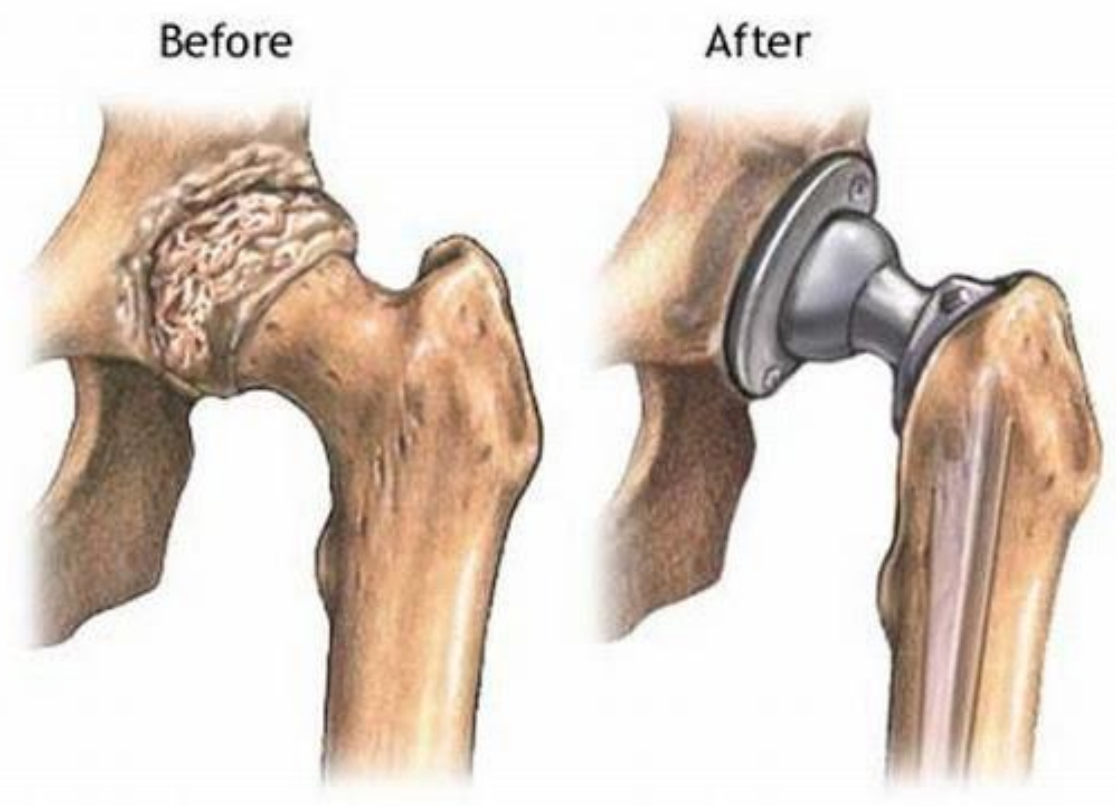

Fig 2: artificial Bones

Artificial Kidney:- Commonly it is mechanical device which cleans the patient blood who is suffering from severe renal diseases or kidney failure. It removes waste products, excess water and salts from human body. Implantable artificial kidney is a bio hybrid device which imitates the function of a healthy kidney. Wearable artificial kidney is a wearable dialysis machine that can be used continuously or daily basis. Various polymers, copolymers, hallow fibers and silicon nanotechnology have been used to fabricate artificial kidney. 


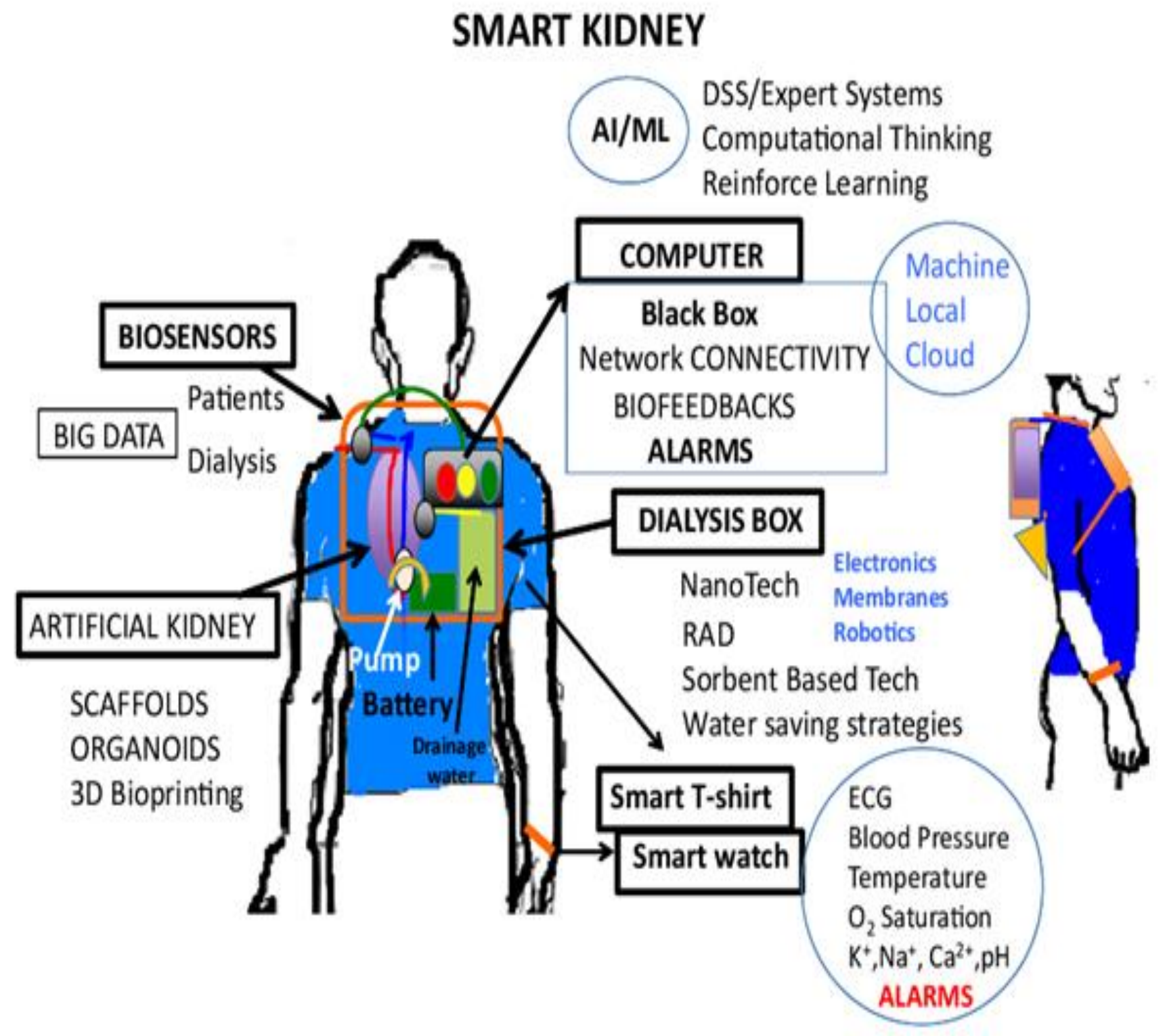

Fig 3: artificial kidney

Artificial Liver:- it is a supportive device which either gives time to liver for regeneration or bridges the patient's liver functions till transplantation. Generally bio artificial livers are bioreactors that are embedded with hepatocytes and these hepatocytes perform normal functions of liver. BAL includes hollow fiber cartridge and the suspension of hepatocytes in gel solution like collagen is injected to hollow fiber matrix. Flat membrane sheet systems are also being developed. 


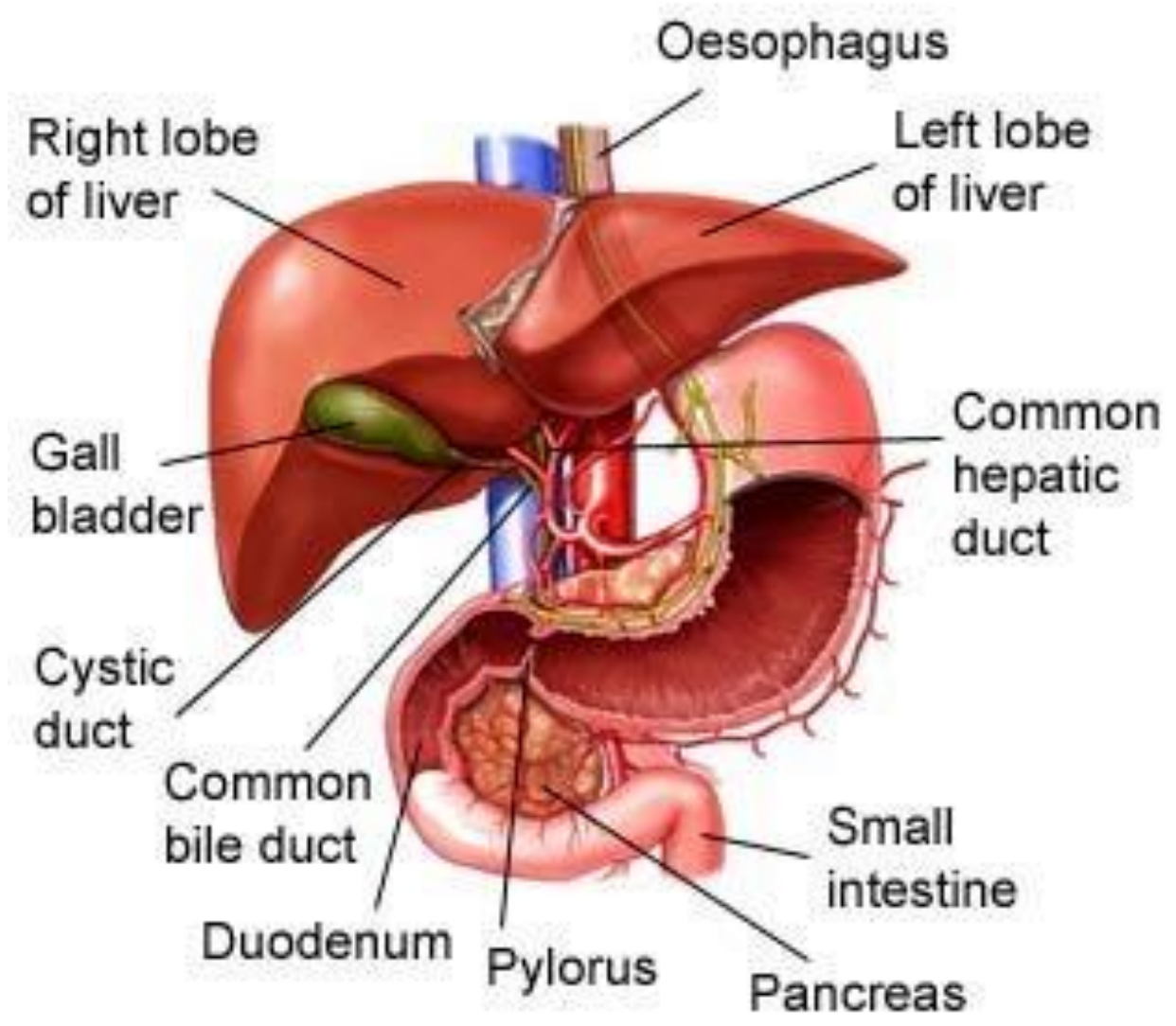

Fig 4: Artificial Liver

\section{CONCLUSION}

In this paper we have discussed about artificial biomaterial. Artificial bio materials are significantly important in the development of artificial organs. Various artificial organs like bone, heart, kidney, liver, lung, pancreas, skin, urinary bladder, auditory brainstem implant, bionic contact lens, cochlear implant, direct acoustic cochlear implant, retinal implant and visual prosthetic parts have been developed.

\section{REFERENCES}

[1] Ross D. Farhadieh, Neil W. Bulstrode \& Sabrina Cugno, Plastic \& Reconstructive Surgery: Approaches \& Techniques, Wiley, 2015.

[2] Charles G. Gebelein, Polymeric Materials \& Artificial organs, ACS Symposium Series, 1984.

[3] Joyce Y. Wong, Joseph D. Bronzino \& Donald R. Peterson, Biomaterials: Principals \& Practice, CRC Press, 2013.

[4] Denise Brehm, Printing Artificial bone, MIT News, June 2013.

[5] Junzo Tanaka \& Toshiyuki Ikoma, Artificial bones for faster bone regeneration, Oct. 2014. 
[6] Rayan R. Joshi, Artificial Heart Research: An Historical Perspective, DASH, 2001.

[7] Anthony Atala, Stuart B. Bauer, Shay Soker, James J. Yoo, Alan B. Retik, The Lancet, 367(9518), 2006, 1241-1246.

[8] Rebecca Zumoff, Implantable Artificial Kidney Project Making Progress, Nephrology News \& Issues, Feb 2016.

[9] Catharine Paddock, Implantable Artificial Kidney based on Microchips sees major progress, Feb 2016.

[10] Gesine Pless, Artificial and Bio-artificial Liver Support, Organogenesis, 3(1), 2007, 20-24.

[11] Palakkan, A. A., Hay, D. C., PR, A. K., TV, K. and Ross, J. A., Liver tissue engineering and cell sources: issues and challenges, Liver International, 33(5), 2013, 666-76.

[12] Heather Nolan, Dongfang Wang \& Joseph B Zwischenberger, Artificial Lung Basics, Organogenesis, 7(1), 2011, 23-27.

[13] William J. Federspiel \& Robert G. Svitek, Lung, Artificial: Current Research and Future Direction, Encyclopedia of Biomaterials and Biomedical Engineering, 2004, 922-931.

[14] Claudio Cobelli, Eric Renard and Boris Kovatchev, Artificial Pancreas: Past, Present, Future, Diabetes, 60(11), 2011, 2672-2682. 